\title{
Antimonotonicity, Crisis and Multiple Attractors in a Simple Memristive Circuit
}

\author{
Christos K. Volos ${ }^{1}$, Akif Akgul' ${ }^{2}$, Viet-Thanh Pham ${ }^{3}$, and Murilo S. \\ Baptista $^{4}$ \\ ${ }^{1}$ Laboratory of Nonlinear Systems - Circuits and Complexity (LANSCOM), \\ Department of Physics, Aristotle University of Thessaloniki, Thessaloniki, \\ GR-54124,Greece,volos@physics.auth.gr \\ ${ }^{2}$ Department of Electrical and Electronics Engineering, Faculty of Technology, \\ Sakarya University, 54187 Serdivan, Sakarya,Turkey, aakgul@sakarya.edu.tr \\ ${ }^{3}$ School of Electronics and Telecommunications, Hanoi University of Science and \\ Technology, 01 Dai Co Viet, Hanoi,Vietnam, thanh.phamviet@hust.edu.vn \\ ${ }^{4}$ Institute for Complex System and Mathematical Biology, University of Aberdeen, \\ Aberdeen, AB243UE,UK, murilo.baptista@abdn.ac.uk
}

\begin{abstract}
In this work a memristive circuit consisting of a first order memristive diode bridge is presented. The proposed circuit is the simplest memristive circuit containing the specific circuitry realization of the memristor to be so far presented in the literature. Characterization of the proposed circuit confirms its complex dynamic behavior, which is studied by using well-known numerical tools of nonlinear theory, such as bifurcation diagram, Lyapunov exponents and phase portraits. Various dynamical phenomena concerning chaos theory, such as antimonotonicity, which is observed for the first time in this type of memristive circuits, crisis phenomenon and multiple attractors have been observed. An electronic circuit to reproduce the proposed memristive circuit was designed, and experiments were conducted to verify the results obtained from the numerical simulations.
\end{abstract}

\section{Introduction}

The discovery of a nanoscale memristor in 2008, [1, 2] at Hewlett-Packard laboratories, that is, a device that was only theoretically postulated in 1971,[3] paved the way to a new research direction. Researchers from different fields 
have focused their attempts to the search of components, whose behavior could be well described by memristors or memristive systems, to the design of new devices with memristive characteristics as well as to the investigation of possible applications, ranging from biological models [4, 5] to adaptive filters $[6]$ and programmable analog integrated circuits. $[7,8]$

Although the physical memristor has been fabricated in 2008, first, it will still be unavailable as a commercial component in the near future because of the cost and the technical difficulties involved in the fabrication of a nanometer-size device. Thus, various memristor emulators, either constructed from off-the-shelf components, $[9,10,11,12,13,14,15]$ or from generalized memristors based on circuits with special topologies, $[9,14,15$, $16,17]$ make greater contributions to modeling, to analyzing, especially to realizing memristor-based chaotic circuits by replacing nonlinear resistance elements in classic chaotic circuits and in this way many novel features of chaotic behaviors could be observed.[18, 19, 20, 21] In more details, several nonlinearities are used to describe the relation between magnetic flux and electric charge of these memristors, such as HP memristor model,[22, 23] non-smooth piecewise linearity,[10, 25] smooth cubic nonlinearity $[12,18,19$, $26]$ and smooth piecewise-quadratic nonlinearity.[18, 25]

Generation of memristor emulators were complicated and not convenient to circuit analysis. However, in 2012, a generalized memristor proposed by Corinto and Ascoli[14] was realized with a full-wave rectifier and a second order RLC filter, which used only elementary electronic circuit elements (diodes, inductor, capacitor and a resistor). This realization has many advantages, such as simple circuit structure, without ground restrictions and being easily accessed to various applications. The key mechanism responsible for the emergence of the memristive behavior is the voltage constraints imposed in each pair of parallel diodes. By replacing the second order RLC filter, in the aforementioned generalized memristor, by a first order parallel RC filter, a new simple first order generalized memristor was proposed by Bao et al.,[28] in 2014, which consists only of six elementary passive elements.

Since then, the specific generalized memristor has been used in a number of published works. Chen et al. were the first who replaced the nonlinear element in a nonlinear circuit with first-order memristive diode bridge.[29] Complex nonlinear phenomena including coexisting bifurcation modes and attractors have been discovered in this circuit. Another interesting memristive chaotic circuit was derived from the classical Chua's circuit by substituting the Chua's diode with the aforementioned generalized memristor.[30] In this circuit the recently discovered new class of attractors, the hidden at- 
tractors, have been observed. Various dynamical phenomena such as chaos and multiple attractors in a memristor-based oscillator obtained from Shinriki's circuit by substituting the nonlinear positive conductance with the first-order memristive diode bridge have been studied by Kengne et al.[31] The complex behavior of a Colpitts chaotic oscillator coupled with the generalized memristor has been investigated in Ref. [32]. Nijitacke et al. studied the coexistence phenomenon of multiple attractors as well as the crisis route to chaos in an autonomous jerk circuit by substituting its nonlinear element with the first-order memristive diode bridge.[33]

In this paper, we propose the simplest memristor-based circuit containing the first-order memristor bridge of Ref. [28], in regard to the aforementioned works [29-33]. The circuit has been designed in such a way that the firstorder memristive diode bridge acts as a nonlinear element. However, innovating from other memristor-based circuits created by replacing an existing nonlinear element with a memristor, it is not based on any known nonlinear system architecture. Circuit's dynamical analysis confirms its complex dynamical behavior and interesting phenomena, such as period-doubling route to chaos, antimonotonicity, interior crisis and coexisting attractor.

The rest of the paper is structured as follows. Section 2 deals with the electronic structure of the generalized memristor as well as with the design of the proposed circuit and its mathematical model, which is derived to investigate the dynamics of the system. The theoretical analysis of system's dynamic characteristics is studied in Section 3. In Section 4 the simulation and experimental results of system's dynamical behavior by using wellknown tools from nonlinear theory, such as bifurcation diagram, Lyapunov exponents and phase portraits are carried out. Finally, the conclusions are summarized in Section 5.

\section{Circuit Description and State Equations}

\subsection{Generalized Memristor}

To begin with, the generalized memristor reported in Ref. [28], is introduced. This circuit consists of a diode bridge with a first order parallel RC filter, as shown in Fig. 1(a), and it is a component of the proposed memristor. Its mathematical model is expressed by the following equations:

$$
\begin{gathered}
i=g\left(v_{C_{m}}, v\right) v=2 I_{S} e^{\left(-\rho v_{C_{m}}\right)} \sinh (\rho v) \\
\frac{d v_{C_{m}}}{d t}=f\left(v_{C_{m}}, v\right)=\frac{2 I_{S} e^{\left(-\rho v_{C_{m}}\right)} \cosh (\rho v)}{C_{m}}-\frac{v_{C_{m}}}{R_{m} C_{m}}-\frac{2 I_{S}}{C_{m}}
\end{gathered}
$$


where $\rho=1 / 2 n V_{T} ; I_{S}, n$ and $V_{T}$ denote the reverse current, the emission coefficient and the thermal voltage of the diode, respectively. [34, 35] Furthermore, $v_{C_{m}}$ is the voltage of the capacitor $C_{m}$, while $v$ and $i$ represent the input voltage and current of the generalized memristor of Fig. 1. With reference to Eq. (1) the generalized memristor is a voltage-controlled and its memductance can be expressed by

$$
G_{M}=\frac{i}{v}=g\left(v_{C_{m}}, v\right)=\frac{2 I_{S} e^{\left(-\rho v_{C_{m}}\right)} \sinh (\rho v)}{v}
$$

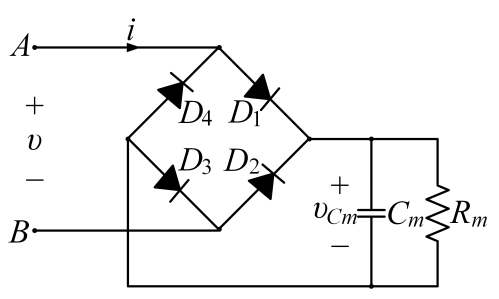

(a)

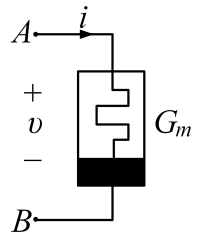

(b)

Figure 1: (a) The generalized memristive diode bridge with the parallel $R C$ filter and (b) the symbol of the generalized memristor $G_{M}$.

The following nominal values of circuit components are used in order to realize the generalized memristive diode bridge: $C_{m}=2 \mu \mathrm{F}, R_{m}=0.5 \mathrm{k} \Omega$ and four 1N4148 diodes with parameters: $I_{S}=1 n A, n=2$ and $V_{T}=26 \mathrm{mV}$.

A sinusoidal voltage source denoted as $v\left(v=V_{m} \sin (\pi f t) V\right)$, is considered to stimulate the memristor in order to study experimentally its behavior. For this reason a digital oscilloscope has been used for capturing the time-series. When $V_{m}=4 V$ and $f$ is set to $200 \mathrm{~Hz}, 1 \mathrm{kHz}$ and $5 \mathrm{kHz}$, respectively, the loci in the $i-v$ plane are hysteresis loops pinched at the origin as shown in Fig. 2(a). As the frequency of the periodic input voltage increases, the area of the pinched hysteresis lobe decreases monotonically and the pinched hysteresis loop shrinks to a nonlinear single-valued function, when the frequency tends to infinity. Furthermore, when $f=200 \mathrm{~Hz}$ and $V_{m}$ is equal to $2 \mathrm{~V}, 4 \mathrm{~V}$ an $6 \mathrm{~V}$, respectivelly, the generalized memristor exhibits the pinched hysteresis loop regardless of the amplitude, as shown in Fig. 2(b). So, according to the results of Ref. [28] the proposed generalized memristor exhibits the three characteristic fingerprints for identifying a memristor.[36] Due to its simplicity, its ease of realization and the absence of ground restrictions the specific circuitry realization of a memristor is an 
attractive candidate for using it in nonlinear circuits as memristor emulator as well as in various memristor's applications.
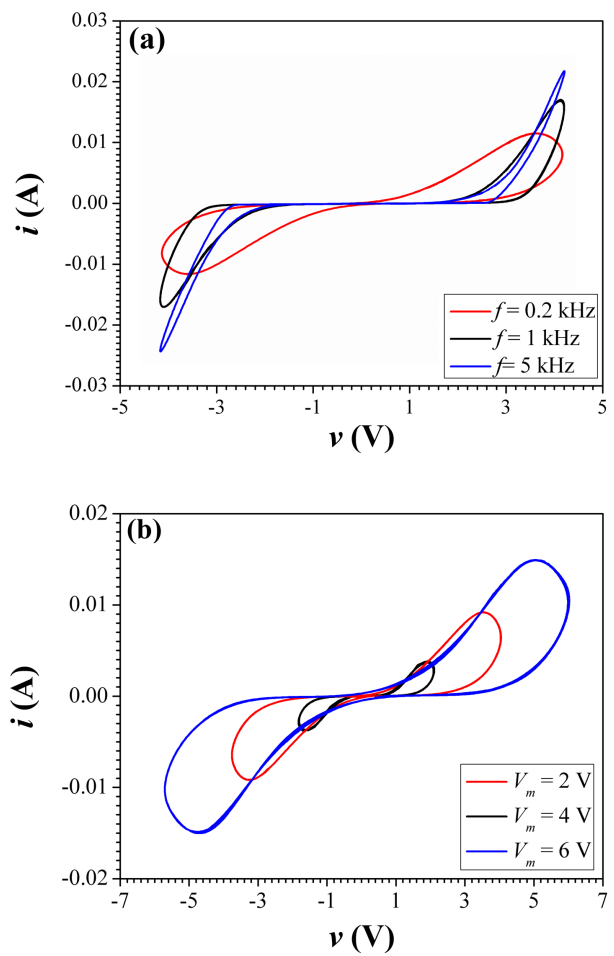

Figure 2: Experimental results of the generalized memristor driven by a periodic input voltage with (a) $V_{m}=4 V$ for different frequencies and (b) $f=200 \mathrm{~Hz}$ for different amplitudes.

\subsection{The Proposed Memristive Circuit}

The schematic diagram of the novel memristive circuit under consideration is depicted in Fig. 3. The circuit consists of resistors, three capacitors and four operational amplifiers (TL084CN), from which three of them $\left(U_{1}-U_{3}\right)$ are configured as integrators, while the fourth $\left(U_{4}\right)$ is used as an inverting amplifier. In this circuit the nonlinear element is the generalized memristor $\left(G_{m}\right)$ of Fig. 1(a).

By applying the Kirchhoff's laws into the proposed circuit of Fig. 3, the 


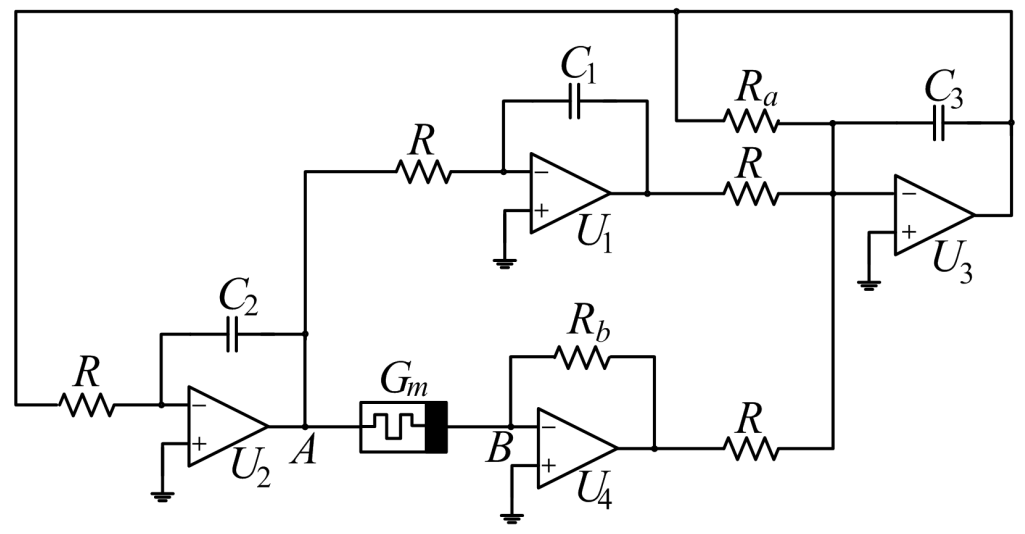

Figure 3: Circuit diagram of the memristive circuit.

following system of differential equations is obtained.

$$
\left\{\begin{array}{l}
C_{1} \frac{d v_{C_{1}}}{d t}=-\frac{1}{R} v_{C_{2}} \\
C_{2} \frac{d v_{C_{2}}}{d t}=-\frac{1}{R} v_{C_{3}} \\
C_{3} \frac{d v_{C_{3}}}{d t}=-\frac{1}{R} v_{C_{1}}-\frac{1}{R_{b}} v_{C_{3}}+\frac{R_{a}}{R} 2 I_{S} e^{\left(-\rho v_{C_{m}}\right)} \sinh \left(\rho v_{C_{2}}\right) \\
C_{m} \frac{d v_{C_{m}}}{d t}=-\frac{1}{R_{m}} v_{C_{m}}+2 I_{S} e^{\left(-\rho v_{C_{m}}\right)} \cosh \left(\rho v_{C_{2}}\right)-2 I_{S}
\end{array}\right.
$$

In the system (4), $v_{C_{1}}, v_{C_{2}}$ and $v_{C_{3}}$ are the voltages across the three capacitors $C_{1}, C_{2}$ and $C_{3}$, respectively. It is noted that these capacitors have the same values $\left(C_{1}=C_{2}=C_{3}=C\right)$ in this work. System (4) is rescaled by using dimensionless variables and parameters given by: $x=\frac{v_{C_{1}}}{n V_{T}}, y=\frac{v_{C_{2}}}{n V_{T}}$, $z=\frac{v_{C_{3}}}{n V_{T}}, w=\frac{v_{C_{m}}}{n V_{T}}, \tau=\frac{t}{R C}, a=\frac{2 R_{a} I_{S}}{n V_{T}}, b=\frac{R}{R_{b}}, c=\frac{2 R I_{S}}{n V_{T}}, d=\frac{R}{R_{m}}$ and $h=\frac{C}{C_{m}}$.

As a result, system (4) is rewritten as:

$$
\left\{\begin{array}{l}
\frac{d x}{d t}=-y \\
\frac{d y}{d t}=-z \\
\frac{d z}{d t}=-x-b z+a e^{-w} \sinh (y) \\
\frac{d w}{d t}=h\left[-d w+c e^{-w} \cosh (y)-c\right]
\end{array}\right.
$$

As seen in (5), the system contains eight terms, one less than the system proposed in Ref. [30]. Also, the circuit of Fig. 3 is the simplest nonlinear circuit based on the generalized memristor of Ref. [28] reported in literature.

The experimental realization of the proposed circuit in Fig. 3 is shown in Fig. 4. The circuit has been built with circuit elements: $R=R a=$ 
$10 k \Omega, R b=16.666 k \Omega, C=10 n F$, while the power supply is $\pm 15 \mathrm{~V}$. In the generalized memristor's realization been used the circuit elements mentioned in Section 2.1.

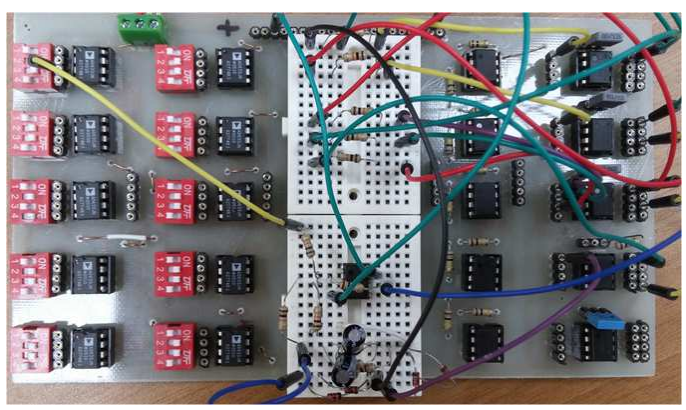

Figure 4: Photograph of the experimental realization of the memristive circuit.

\section{Theoretical Analysis of System's Characteristics}

In this Section the theoretical analysis of the proposed system (5), by studying well-known characteristics of dynamical systems, such as dissipation, invariance and symmetry, is presented.

\subsection{Dissipation and Invariance}

According to system (5), the divergence of the system is

$$
\nabla V=\frac{\partial \dot{x}}{\partial x}+\frac{\partial \dot{y}}{\partial y}+\frac{\partial \dot{z}}{\partial z}+\frac{\partial \dot{w}}{\partial w}=-b-d h-\operatorname{ch} e^{-w} \cosh (y)
$$

where $V$ indicates the phase volume. As the function $e^{-w} \cosh (y)>0$, then $\nabla V<0$, for every $y$ and $w$, so the state change of the system is bounded, therefore system (5) is dissipative. So, it converges in the index of the following form:

$$
\frac{d V}{d t}=e^{\left[-b-d h-c h e^{-w} \cosh (y)\right] t}
$$

That is, in the system (5), the time rate of change of phase volume is $e^{\left[-b-d h-c h e^{-w} \cosh (y)\right] t}$, a volume element $V_{0}$ is apparently contracted by the flow into a volume element $V_{0} e^{\left[-b-d h-c h e^{-w} \cosh (y)\right] t}$ at time $t$.

This means that each volume containing the trajectories of system (5) will shrinks to zero when $t \rightarrow \infty$ with the exponential rate $V_{0} e^{\left[-b-d h-c h e^{-w} \cosh (y)\right] t}$. 
Thus, all the orbits of the system (5) are finally confined to a specific subset of zero volume, and the asymptotic motion of system (5) will be fixed on an attractor of the system.

\section{$3.2 \quad$ Symmetry}

The equilibrium points of system (5) can be found by solving

$$
\left\{\begin{array}{l}
-y=0 \\
-z=0 \\
-x+a e^{-w} \sinh (y)-b z=0 \\
h\left[-d w+c e^{-w} \cosh (y)-c\right]=0
\end{array}\right.
$$

Thus, system (5) has a single equilibrium point $E(0,0,0,0)$. In general, the number of equilibrium points is not equal to the order of the system, that is, the number of the equilibrium points is independent of the order of the system. To linearize the system shown by system (5) at the equilibrium point $E$, the corresponding Jacobian matrix is expressed by

$$
J_{E}=\left[\begin{array}{cccc}
0 & -1 & 0 & 0 \\
0 & 0 & -1 & 0 \\
-1 & a & -b & 0 \\
0 & 0 & 0 & -h(c+d)
\end{array}\right]
$$

Therefore, the characteristic equation of system (5) at the equilibrium point $E$ can be found as:

$$
\begin{aligned}
& \left|\lambda I_{d}-J_{E}\right|=\lambda^{4}+[b+h(c+d)] \lambda^{3}+[a+b h(c+d)] \lambda^{2} \\
& +[1+a h(c+d)] \lambda+h(c+d)=0
\end{aligned}
$$

The eigenvalues can be obtained from Eq. (10) to determine the stability of this equilibrum point. Obviously, the stability of the equilibrium point of system (5) depends on system parameters. By choosing $a=c=3.846 \cdot 10^{-4}$, $b=0.6, d=20$ and $h=5 \cdot 10^{-3}$, and by using the circuit's elements: $R=R_{a}=10 \mathrm{k} \Omega, R_{b}=16.666 \mathrm{k} \Omega$ and $C=10 \mathrm{nF}$, the eigenvalues are: $\lambda_{1}=-1.24492, \lambda_{2}=-0.1, \lambda_{3}=0.322461-0.836231 i$ and $\lambda_{4}=0.322461$ $+0.836231 i$. So $E$ is a saddle focus equilibrium point of index 2. For many chaotic systems, such as the well-known Chua's circuit, saddle focus equilibrium point of index 2 is the premise of scroll motion. 

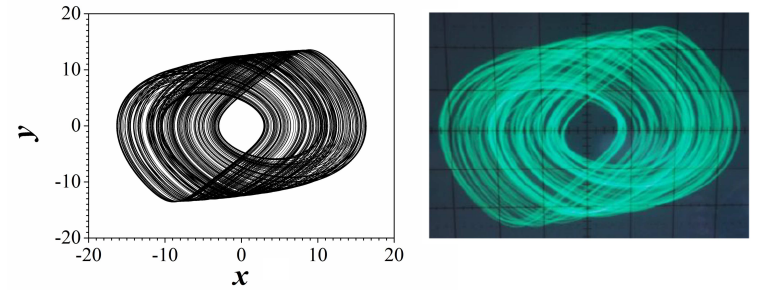

(a)

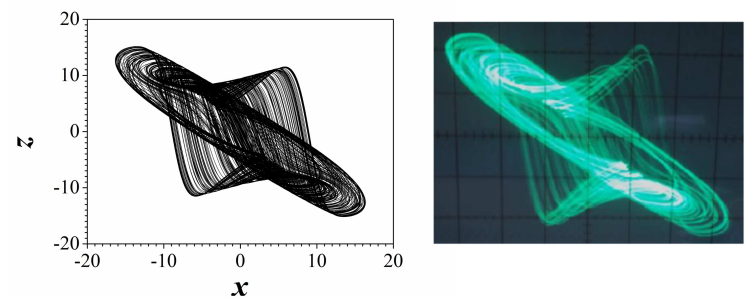

(b)
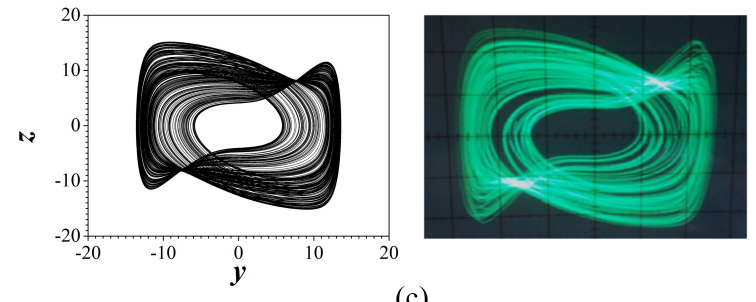

(c)

Figure 5: Simulation and experimental chaotic attractor of system (5) for $a=c=3.846 \cdot 10^{-4}, b=0.6, d=20$ and $h=5 \cdot 10^{-3}$ in (a) $x-y$ plane, (b) $x-z$ plane, and (c) $y-z$ plane.

\section{Dynamical Properties of the Circuit}

In this section the analysis of the proposed memristive circuit's dynamical behavior with the aforementioned set of system's (5) parameters and initial conditions $\left(x_{0}, y_{0}, z_{0}, w_{0}\right)=(0,0.1,0,0.2)$, by using well-known tools of nonlinear theory, such as phase portrait, bifurcation diagram and Lyapunov spectrum, is performed. For the investigation of the proposed memristive circuit's dynamical behavior, system (5) is integrated numerically using the classical fourth-order Runge-Kutta integration algorithm. For each set of parameters used in this work, the time step is always $\Delta t=0.002$ and the calculations are performed using variables and parameters in extended precision mode. For each parameter settings, the system is integrated for a 
sufficiently long time and the transient is discarded. Furthermore, the calculated Lyapunov exponents of system (5), for the chosen set of parameters and initial conditions, by using the Wolf et al. algorithm,[37] are: $L_{1}=$ $0.2374, L_{2}=0, L_{3}=-0.15566$ and $L_{4}=-1.14209$. In this case, system (5) is chaotic because it has one positive Lyapunov exponent. Simulation and experimental chaotic attractors of the memristive circuit are displayed in Fig. 5 .

To study the type of scenario giving rise to chaos by considering the resistor $R_{b}$, namely the parameter $b$ in system (5), as the main bifurcation control parameter, the bifurcation diagram in Fig. 6 is obtained, while the other parameters remain fix. The bifurcation diagram is obtained by plotting the variable $x$ when the trajectory cuts the plane $y=0$ with $d y / d t<$ 0 , as the control parameter $b$ is increased (or decreased) in tiny steps in the range $0.2 \leq b \leq 1.0$. Also, the final state for a value of the control parameter serves as the initial state for the next iteration. This procedure carried out in our numerical simulations reproduces more closely the experimental study of circuit's dynamical behavior by using a variable resistor as $R_{b}$. In the graph of Fig. 6(a), two set of data corresponding respectively for increasing (black) and decreasing (red) values of $b$ are superimposed. This strategy represents a simple way to localize the range of values in which the system develops coexisting behavior. Furthermore, the corresponding spectrum of the three largest Lyapunov exponents is shown in Fig. 6(b). It can be seen that the bifurcation diagram well coincide with the spectrum of the Lyapunov exponents.

In more details, from the bifurcation diagrams of Fig. 6(a) it is possible to verify coexistence of attractors in the system (5) for the range of $0.2 \leq b \leq 0.4$. In this region the system enters to chaos through a period doubling route with the same initial period-1 steady state but beginning from different values of $x$, for $b=0.2$. This coexistence is a consequence of the symmetry in the system and it depends on the fact that different initial conditions in phase space leading to different attractors. [38, 39, 40, 41] In Fig. 7 the experimental coexisting chaotic attractors at $b=0.35$ is depicted. For $b>0.4$ the coexisting attractors disappear and the system through an interior crisis[42] is driven to an extended chaotic attractor, as it is observed in the bifurcation diagrams of Fig. 6(a). The occurrence of sudden qualitative changes of chaotic dynamics as a parameter is varied is known from 1983. That year Grebogi et al. reported that such changes may result from the collision of an unstable periodic orbit and a coexisting chaotic attractor. These phenomena was called crises. Since then crises phenomena and especially the interior crisis, which is one of the crises types have been observed 

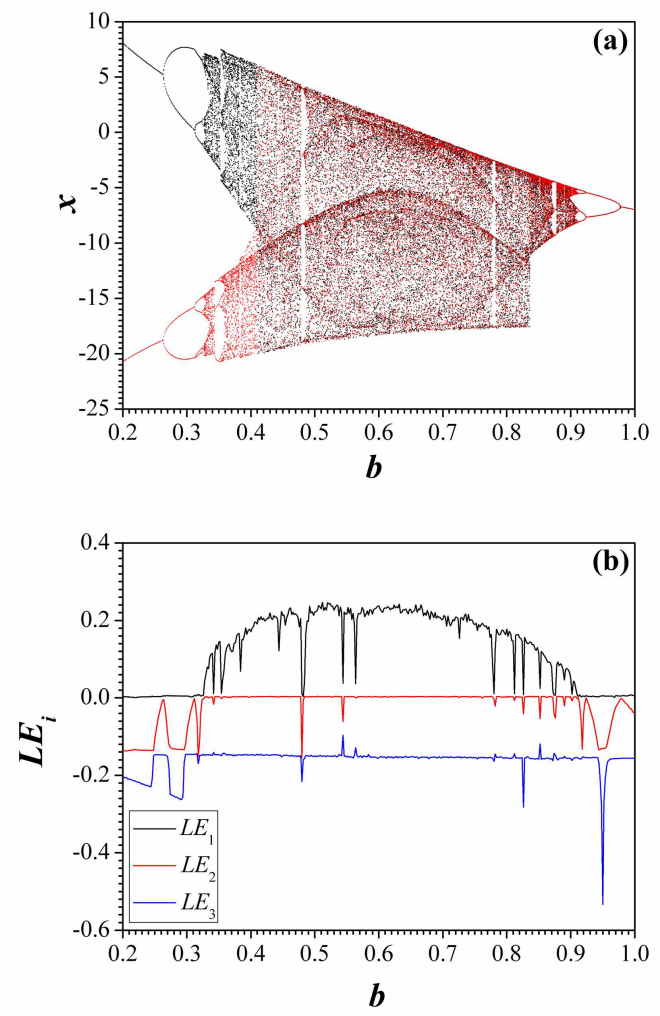

Figure 6: (a) Bifurcation diagram of system (5) for increasing (black) and decreasing (red) values of $b$ and (b) the corresponding graph of the three largest Lyapunov exponents plotted in the range $0.2 \leq b \leq 1.0$.

in many dynamical systems. [43, 44, 45, 46]

Also, with a more clear view in the bifurcation diagrams of Fig. 6(a) a second interesting phenomenon concerning system's (5) dynamical behavior can be observed. This is the antimonotonicity, which has been introduced by Dawson et al..[47] This is a fundamental phenomenon in bifurcations for a large class of nonlinear dissipative systems, $[48,49,50]$ where periodic orbits not only created but also destroyed, when one increases the control parameter monotonically (smoothly) in any neighborhood of a homoclinic tangency value-inevitable reversals of period doubling cascades. So, antimonotonicity is due to tangential intersections between the stable and unstable manifolds of a system.

According to this phenomenon, the system enters to chaos with the well- 
known period doubling route $(\mathrm{p}-1 \rightarrow \mathrm{p}-2 \rightarrow \ldots \rightarrow$ chaos $)$ and exits from the chaos following the reverse period doubling route $(\operatorname{chaos} \rightarrow \ldots \rightarrow \mathrm{p}-2 \rightarrow \mathrm{p}-1)$. As a result, a shape of a chaotic bubble is formed in the bifurcation diagram. This phenomenon is relevant because it describes a complex scenario of how a nonlinear system creates or destroys unstable periodic orbits by parameter alterations. At the parameter value where the Lyapunov exponent is maximal, the chaotic attractor has embedded on it the largest amount of unstable periodic orbits that the dynamics can create. This observation is the first of its kind to be reported in a circuit based on the generalized memristive diode-bridge circuit with parallel $R C$ filter.
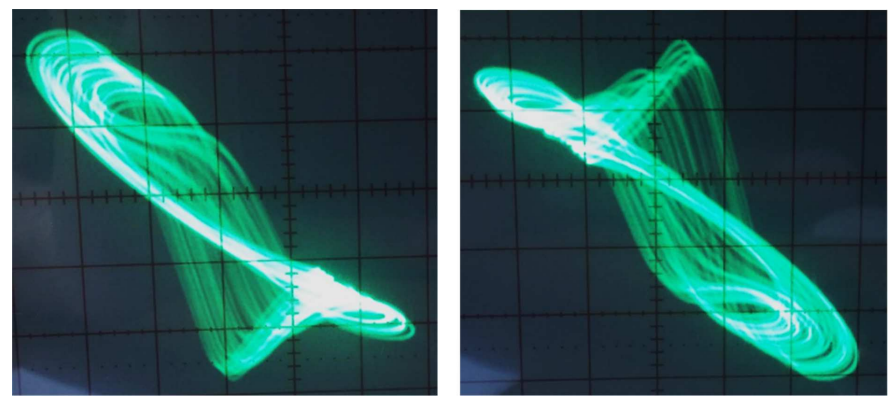

Figure 7: Experimental coexisting chaotic attractors, for $b=0.35$.

\section{Conclusion}

A memristive circuit containing a first order memristive diode bridge was presented in this paper. The dynamics of the introduced system can be strongly chaotic presenting interesting phenomena, such as period doubling route to chaos, antimonotonicity, interior crisis and coexisting attractors. Especially, the phenomenon of antimonotonicity is observed for the first time in this type of memristive circuits with a first order memristive diode bridge. Simulation results by using well-known tools from nonlinear theory, such as bifurcation diagram, Lyapunov exponents and phase portraits confirmed the appearence of the aforementioned phenomena. An electronic circuit to reproduce the proposed memristive circuit was designed, and experiments were conducted to verify the results obtained from the numerical simulations. Circuits with coexistence of attractors and interior crisis can be used to understand tipping points[51]. Those circuits that present antimonotonicity and are simultaneously simple in electronic architecture are specially interesting to construct electronic based circuits for chaos-based 
cryptography with expanded key spaces, [52] or to create fast and light (low computation power required) chaos-based cryptosystems, such as in Ref. [53], or to create classical crytosystems that inherit the quantum cryptographic property of detecting the eavesdropper.[54] Such a system can be used in potential chaos-based applications as well in the understanding of the memristive systems' dynamical behavior. However, the advantages of the proposed circuit in real engineering applications will be further explored in future work.

\section{References}

[1] D. B. Strukov, G. S. Snider, D. R. Stewart and R. S. Williams, The missing memristor found, Nature 453 (2008) 80-83.

[2] J. M. Tour and T. He, Electronics: the fourth element, Nature 453 (2008) 42-43.

[3] L. Chua, Memristor-the missing circuit element, IEEE Transactions on Circuit Theory 18 (1971) 507-519.

[4] Y. V. Pershin, S. La Fontaine and M. Di Ventra, Memristive model of amoeba learning, Physical Review E 80(2) (2009) 021926.

[5] M. J. Sharifi and Y. M. Banadaki, General spice models for memristor and application to circuit simulation of memristor-based synapses and memory cells, Journal of Circuits, Systems, and Computers 19(02) (2010) 407-424.

[6] T. Driscoll, J. Quinn, S. Klein, H. T. Kim, B. J. Kim, Y. V. Pershin, M. Di Ventra and D. N. Basov, Memristive adaptive filters, Applied Physics Letters 97(9) (2010) 093502.

[7] S. Shin, K. Kim and S. M. Kang, Memristor applications for programmable analog ICs, IEEE Transactions on Nanotechnology 10(12) (2011) 266-274.

[8] L. Chua, Resistance switching memories are memristors, Applied Physics A 102(4) (2011) 765-783.

[9] H. Kim, M. P. Sah, C. J. Yang, S. Cho and L. O. Chua, Memristor emulator for memristor circuit applications, IEEE Transactions on Circuits and Systems I: Regular Papers 59 (2012) 2422-2431. 
[10] B. Bo-Cheng, X. Jian-Ping, Z. Guo-Hua, M. Zheng-Hua and Z. Ling, Chaotic memristive circuit: Equivalent circuit realization and dynamical analysis, Chinese Physics B 20(12) (2011) 120502.

[11] C. Sanchez-Lopez and J. Mendoza-Lopez, A floating analog memristor emulator circuit, IEEE Transactions on Circuits and System II: Express Briefs 561 (2014) 309-313.

[12] B. Muthuswamy, Implementing memristor based chaotic circuits, International Journal of Bifurcation and Chaos 20(5) (2010) 1335-1350.

[13] X. Y. Wang, A. L. Fitch, H. H. C. Iu, V. Sreeram and W. G. Qin, Implementation of an analogue model of a memristor based on a lightdependent resistor, Chinese Physics B 21 (2012) 108501.

[14] F. Corinto and A. Ascoli, Memristive diode bridge with LCR filter, Electronics Letters, 48 (2012) 824-825.

[15] X. Wang, G. Wang and X. Wang, X, Dynamic character analysis of a LDR, memristor-based chaotic system, Journal of Circuits, Systems, and Computers 23(06) (2014) 1450085.

[16] B. C. Bao, J. J. Yu, F. W. Hu and Z. Liu, Generalized memristor consisting of diode bridge with first order parallel RC filter, International Journal of Bifurcation and Chaos 24 (2014) 14501432.

[17] B. C. Bao, Z. Liu and J. P. Xu, Steady periodic memristor oscillator with transient chaotic behaviors, Electronics Letters 46 (2010) 228-230.

[18] H. H. C. Iu, D. S. Yu, A. L. Fitch, V. Sreeram and H. Chen, Controlling chaos in a memristor based circuit using a twin-T notch filter, IEEE Transactions on Circuits and Systems I 58 (2011) 1337-1344.

[19] Z. G. Cam and H. Sedef, A new floating memristance simulator circuit based on second generation current conveyor, Journal of Circuits, Systems and Computers 26(02) (2017) 1750029.

[20] B. C. Bao, J. P. Xu and Z. Liu, Initial state dependent dynamical behaviors in memristor based chaotic circuit, Chinese Physics Letters 27 (2010) 070504.

[21] A. L. Fitch, D. Yu, H. H. C. Iu and V. Sreeram, Hyperchaos in a memristor-based modified canonical Chua's circuit, International Journal of Bifurcation and Chaos 22 (2012) 1250133. 
[22] G. Y. Wang, J. L. He, F. Yuan and C. J. Peng, Dynamical behaviors of a TiO2 memristor oscillator, Chinese Physics Letters 30(2) (2013) 110506 .

[23] L. Wang, E. Drakakis, S. Duan, P. He and X. Liao, Memristor model and its application for chaos generation, International Journal of Bifurcation and Chaos 22 (2012) 1250205.

[24] B. Muthuswamy and P. P. Kokate, Memristor-based chaotic circuits, IETE Techical Review 26 (2009) 415-426.

[25] H. Sozen and U. Cam, First-order memristor-capacitor filter circuits employing HP memristor, Journal of Circuits, Systems, and Computers 23(08) (2014) 1450116.

[26] M. Itoh and L. O. Chua, Memristor oscillators, International Journal of Bifurcation and Chaos 18 (2008) 3183-3206.

[27] B. C. Bao, Z. H. Ma, J. P. Xu, Z. Liu and Q. Xu, A simple memristor chaotic circuit with complex dynamics, International Journal of Bifurcation and Chaos 21 (2014) 2629-2645.

[28] B. Bao, J. Yu, F. Hu and Z. Liu, Generalized memristor consisting of diode bridge with first order parallel RC filter, International Journal of Bifurcation and Chaos 24 (2014) 1450143.

[29] M. Chen, J. Yu, Q. Yu, C. Li and B. Bao, A memristive diode bridgebased canonical Chua's circuit, Entropy, 16(12) (2014) 6463-6476.

[30] M. Chen, M. Li, Q. Yu, B. Bao, Q. Xu and J. Wang, Dynamics of selfexcited attractors and hidden attractors in generalized memristor-based Chua's circuit, Nonlinear Dynamics 81(1-2) (2015) 215-226.

[31] J. Kengne, Z. N. Tabekoueng, V. K., Tamba and A. N. Negou, Periodicity, chaos, and multiple attractors in a memristor-based Shinriki's circuit, Chaos: An Interdisciplinary Journal of Nonlinear Science 25(10) (2015) 103126.

[32] L. Lu, C. Li, Z. Zhao, B. Bao and Q. Xu, Colpitts Chaotic oscillator coupling with a generalized memristor, Mathematical Problems in Engineering 2015 (2015) Article ID 249102, 9 pages, 2015.

[33] Z. T. Njitacke, H. B. Fotsin, A. N. Negou and D. Tchiotsop, Coexistence of multiple attractors and crisis route to chaos in a novel memristive 
diode bridge-based Jerk circuit, Chaos, Solitons and Fractals 91 (2016) 180-197.

[34] M. P. Hanias, G. Giannaris and A. R. Spyridakis, Time series analysis in chaotic diode resonator circuit, Chaos, Solitons and Fractals 27 (2006) 569-573.

[35] D. W. Sukov, M. E. Bleich, J. Gauthier and J. E. S. Socolar, Controlling chaos in a fast diode resonator using extended time-delay autosynchronization: Experimental observations and theoretical analysis, Chaos 7(10) (1997) 560-576.

[36] S. P. Adhikari, M. P. Sah, H. Kim and L. Chua, Three fingerprints of memristor, IEEE Transactions on Circuits and Systems I 60(11) (2013) 3008-3021.

[37] S. A. Wolf, J. B. Swift, H. L. Swinney and J. A. Vastano, Determining Lyapunov exponents from a time series, Physica D: Nonlinear Phenomena 16(3) (1985) 285-317.

[38] H. Kantz and T. Schreiber, Nonlinear time series analysis (Vol. 7), Cambridge University Press, Cambridge (2004).

[39] P. M. Battelino, C. Grebogi, E. Ott, J. A. Yorke and E. D. Yorke, Multiple coexisting attractors, basin boundaries and basic sets, Physica D: Nonlinear Phenomena, 32(2), (1988) 296-305.

[40] D. K. Bandy, L. M. Narducci and L. A. Lugiato, Coexisting attractors in a laser with an injected signal, JOSA B, 2(1),(1985) 148-155.

[41] S. Banerjee, Coexisting attractors, chaotic saddles, and fractal basins in a power electronic circuit, IEEE Transactions on Circuits and Systems I: Fundamental Theory and Applications, 44(9), (1997) 847-849.

[42] R. W. Rollins and E. R. Hunt, Intermittent transient chaos at interior crises in the diode resonator, Physical Review A 29(6) (1984) 3327.

[43] R. W. Rollins and E. R. Hunt, Intermittent transient chaos at interior crises in the diode resonator, Physical Review A, 29(6), (1984) 33273335 .

[44] C. Grebogi, E. Ott and J. A. Yorke, Crises, sudden changes in chaotic attractors and transient chaos, Physica D: Nonlinear Phenomena, 7, (1983) 181-200. 
[45] A. Witt, U. Feudel and A. Pikovsky, Birth of strange nonchaotic attractors due to interior crisis, Physica D: Nonlinear Phenomena, 109, (1997) 180-190.

[46] J. M. Gonzlez-Miranda, Observation of a continuous interior crisis in the Hindmarsh-Rose neuron model, Chaos: An Interdisciplinary Journal of Nonlinear Science, 13(3), (2003) 845-852.

[47] S. P. Dawson, C. Grebogi, J. A. Yorke, I. Kan and H. Kocak, Antimonotonicity: inevitable reversals of period-doubling cascades, Physics Letters A 163(3) (1992) 249-254.

[48] I. Kan and J. A. Yorke, Antimonotonicity: Concurrent creation and annihilation of periodic orbits, Bulletin of the American Mathematical Society, 23(2), (1990) 469-476.

[49] L. Kocarev, K. S. Halle, K., Eckert and L. O. Chua, Experimental observation of antimonotonicity in Chua's circuit, International Journal of Bifurcation and Chaos, 3, (1993) 1051-1055.

[50] I. M. Kyprianidis, I. N. Stouboulos, P. Haralabidis and T. Bountis, Antimonotonicity and chaotic dynamics in a fourth-order autonomous nonlinear electric circuit, International Journal of Bifurcation and Chaos, 10, (2000) 1903-1915.

[51] E. S. Medeiros, I. L. Caldas, M. S. Baptista and U. Feudel, Trapping phenomenon attenuates tipping points for limit cycles, Scientific Reports (2017), in press.

[52] M. Farajallah, S. El Assad and O. Deforges, Fast and secure chaosbased cryptosystems for images, International Journal of Bifurcation and Chaos, 26, (2016) 1650021.

[53] G. Vidal, M. S. Baptista and H. Mancini, A fast and light stream cipher for smartphones, Europhysics Journal Special Topic, 223, (2014) 16011610 .

[54] G. Vidal, M. S. Baptista and H. Mancini, Fundamentals of a classical chaos-based crypto system with some quantum cryptography features, International Journal of Bifurcation and Chaos, 22, 1250243 (2012). 